\title{
Load-Dependent Frictional Performance of Vitamin E-Blended Ultrahigh Molecular Weight Polyethylene in Serum Lubricant
}

\author{
Yasushi Okubo $^{1)}$, Daisuke Hamada ${ }^{1}$, Koji Yamamoto ${ }^{1)}$, Shin-ichiro Mori ${ }^{1}$, \\ Ken Ikeuchi $^{2}$ and Naohide Tomita ${ }^{1)^{*}}$ \\ ${ }^{1)}$ Graduate School of Engineering, Kyoto University \\ Yoshida-Honmachi, Sakyo-ku, Kyoto 606-8501, Japan \\ ${ }^{2)}$ Department of Clinical Engineering, Suzuka University of Medical Science \\ 1001-1, Kishioka-cho, Suzuka, Mie 510-0293, Japan \\ *Corresponding author: ntomita@iic.kyoto-u.ac.jp
}

( Manuscript received 17 March 2010; accepted 12 April 2010; published 30 April 2010 )

\begin{abstract}
The load-dependent frictional performance of vitamin E-blended ultrahigh molecular weight polyethylene (UHMWPE) in serum lubricant was examined. The UHMWPE specimens were slid against the surface of Co-28Cr-6Mo alloy by using a pin-on-disk friction test apparatus. The frictional performance of vitamin E-blended UHMWPE differed from that of virgin UHMWPE under $30 \mathrm{MPa}$ loading, while little difference was observed under $3 \mathrm{MPa}$ loading. The friction force of vitamin E-blended UHMWPE showed a lower value in initial friction and a higher value in steady state friction, and the variability of friction force in the transition process was relatively small. Frictional performance similar to that of the vitamin E-blended UHMWPE was observed as a result of applying vitamin $\mathrm{E}$ to the sliding surface of virgin UHMWPE. These results suggest that the frictional performance of vitamin E-blended UHMWPE in serum lubricant is affected by the presence of vitamin $\mathrm{E}$ on the sliding surface under high compressive load.
\end{abstract}

Keywords: artificial knee joint, UHMWPE, vitamin E, wear, friction, pressure leaching, serum proteins

\section{Introduction}

Ultrahigh molecular weight polyethylene (UHMWPE) is commonly used as a sliding surface in total joint prostheses. However, UHMWPE implants impose limitations on the lifetime of artificial knee joints as a result of delamination fracture and wear ${ }^{1,2)}$. To address this issue, UHMWPE blended with vitamin $\mathrm{E}$ ( $d l-\alpha$-Tocopherol) has been developed in order to prevent delamination fracture, which is a type of fatigue failure accelerated by oxidation and often observed in UHMWPE knee components $^{3)}$. Vitamin E-blended UHMWPE has been reported to prevent crack initiation at subsurface grain boundaries of UHMWPE under multidirectional deformation ${ }^{4}$. On the other hand, it is currently believed that osteolysis and subsequent aseptic loosening are thought to arise from a biological response to debris from UHMWPE wear ${ }^{5,6)}$. Therefore, the wear performance of UHMWPE and the biological reactivity to wear debris are important issues for improving the longevity of knee prostheses. It has been reported that the wear volume of vitamin E-blended UHMWPE, as tested with a knee joint simulator, is approximately $30 \%$ lower than that of virgin UHMWPE at 5 million cycles ${ }^{7}$. It has also been reported that cells cultured with debris from vitamin E-blended UHMWPE secrete significantly lower quantities of inflammatory cytokines compared with those cultured with debris from virgin UHMWPE $^{8}$. However, detailed mechanisms for these phenomena have not yet been clarified.

In order to clarify the effects of vitamin $\mathrm{E}$ on the wear resistance mechanism of UHMWPE, investigations have been carried out considering the bulk material and its surface properties. Based on the bulk structure of vitamin E-blended UHMWPE, it has been suggested that vitamin $\mathrm{E}$ in the amorphous phase of UHMWPE decreases strain-induced crystallization and increases strain-induced orientation of molecular chains in the amorphous phase ${ }^{9)}$. It has also been suggested that the prevention of oxidation chain reactions (radical reactions) by vitamin E might alter the performance of $\gamma$-irradiated UHMWPE ${ }^{1)}$. Considering surface phenomena such as solid-solid and solid-liquid interactions, we have confirmed the possibility of the pressure-induced leaching of vitamin $\mathrm{E}$ from inside of 
vitamin E-blended UHMWPE ${ }^{10)}$. In regard to the solid-solid interaction, we have suggested that vitamin $\mathrm{E}$ reduces the adhesion between the surfaces of UHMWPE and Co-28Cr-6Mo alloy ${ }^{11)}$. In the present study, we focused on solid-liquid interactions and examined the frictional performance of vitamin E-blended UHMWPE in serum lubricant.

\section{Materials and Methods}

\subsection{Preparation of specimens}

UHMWPE resin powder (GUR1050, Ticona, USA) was blended with vitamin E $(0.3$ mass\%, $d l$ - $\alpha$-Tocopherol, Eisai, Japan) using a screw cone mixer (LFS-GS-2J, Fukae Powtec, Japan). The vitamin E-blended UHMWPE block was manufactured by direct compression molding at $220^{\circ} \mathrm{C}$ and $25 \mathrm{MPa}$ for $30 \mathrm{~min}$. The virgin UHMWPE block, which was used as the control material in this study, was manufactured similarly, but without the addition of vitamin E. The pin specimens for the friction tests were machined from these UHMWPE blocks. The pin geometry was a flat-ended conical cylindrical shape $(\phi 5 \times 5 \mathrm{~mm})$ with a tip diameter of $\phi 1 \mathrm{~mm}\left(R_{a}<0.1 \mu \mathrm{m}\right)$. Then, pin specimens were subjected to ultrasonic immersion cleaning in isopropyl alcohol (50 vol\%) at room temperature for $15 \mathrm{~min}$. In addition, disk specimens with a highly polished flat sliding surface and diameter of $8 \mathrm{~mm}\left(R_{a}<0.01 \mu \mathrm{m}\right)$ were machined from a Co-28Cr-6Mo alloy ingot and cleaned by ultrasonication in acetone $(99.5 \mathrm{vol} \%)$ at room temperature for $15 \mathrm{~min}$. All manufacturing and cleaning procedures were conducted in air, and no sterilization was carried out prior to the testing.

\subsection{Pin-on disk friction test}

Friction tests were carried out using a computer-controlled pin-on-disk tribological test apparatus (Ys-TRIB-01, Sanki, Japan), as shown in Fig. 1. The pin specimen was mounted vertically at the tip of the leaf spring. A load of $2.4 \mathrm{~N}$ or $24 \mathrm{~N}$ was applied to the pin specimen; the nominal contact stress on the pin surface was $3 \mathrm{MPa}$ or $30 \mathrm{MPa}$, respectively. The disk specimen was fixed within the lubricant bath on the $\mathrm{X}$-stage, and set into linear reciprocating sliding motion for 2,000 cycles with amplitude of $1 \mathrm{~mm}$ and frequency of $0.3 \mathrm{~Hz}$. The lubricant bath was filled with $5 \mathrm{ml}$ of bovine calf serum (SAFC Biosciences, USA), which was kept at $37^{\circ} \mathrm{C}$ and replenished at a rate of $1 \mathrm{ml}$ per 500 cycles during the tests to compensate for its gradual evaporation.

\subsection{Application of vitamin $\mathrm{E}$ to pin surface}

Vitamin E solution $(30 \mu \mathrm{g} / \mathrm{ml})$ was prepared by dissolving vitamin $\mathrm{E}$ in ethanol (99.5 vol\%), and $0.5 \mu \mathrm{l}$ of solution was placed on the tip of the virgin pin specimen. After evaporation of ethanol, the amount of vitamin $\mathrm{E}$ residue on the tip of the pin specimen was about $1.5 \mu \mathrm{g} / \mathrm{cm}^{2}$. Next, the pin specimen was mounted on the leaf spring, and the friction test was carried out.

\subsection{Definition of friction force}

The friction force between the UHMWPE pin and the Co-28Cr-6Mo alloy disk was calculated from the displacement of the leaf spring during the sliding motion. A typical example of the output signal of the eddy-current displacement sensor during friction force measurements is shown in Fig. 2. The positive and negative friction forces indicate the opposite sliding directions during the linear reciprocating motion. The friction force was defined as the average value of dynamic friction force within the central area of $1 \mathrm{~mm}$ on the sliding trajectory.

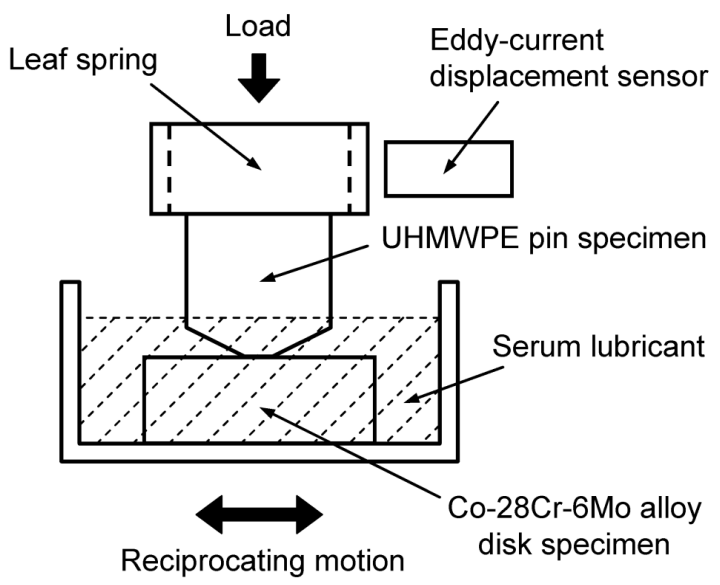

Fig. 1 Schematic diagram of experimental apparatus for friction test

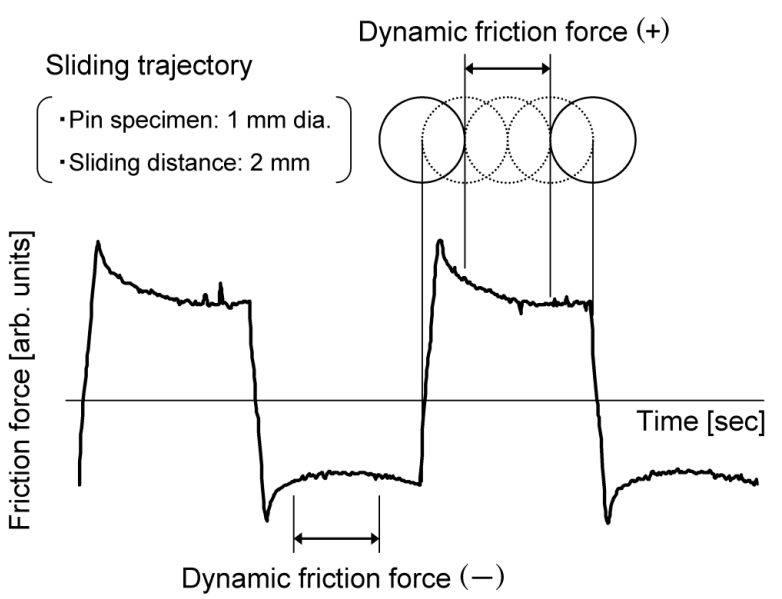

Fig. 2 Typical example of output signal from eddy-current displacement sensor during friction force measurement between UHMWPE pin and Co-28Cr-6Mo alloy disk 


\subsection{Quantification of serum-derived residue}

After friction tests, the Co-28Cr-6Mo alloy disk specimens were cleaned by immersion in ultrapure water and then dried at room temperature inside a clean bench over night. The actual micrograph of the Co-28Cr-6Mo alloy surface after the friction test is shown in Fig. 3. A considerable amount of residue was distributed over the entire sliding region on the Co-28Cr-6Mo alloy surface. Given the experimental conditions, it is concluded that these residues were substance derived from serum proteins. The quantification process for serum-derived residues on the disk specimen surface is shown in Fig. 4. The tested disk specimen was observed under a digital microscope (VHX-900, Keyence, Japan) at 300-fold magnification and photographed as an 8-bit grayscale image. An image of the central part of the sliding region $(0.5 \times 0.5$ $\mathrm{mm}^{2}$ at a resolution of $500 \times 500$ pixels) was acquired, and all pixels were classified in accordance with their brightness. The histogram took the form of a bell-shaped curve with a more gradual slope in the low brightness region than in the high brightness region. This slightly more gradual slope was assumed to be the result of the serum-derived residues produced by reciprocating sliding of the specimens, and is thus defined as the apparent residue. The apparent residue region and the background region were digitalized using a threshold value of brightness. The threshold value for the brightness histogram was set as the value at the opposite side of the maximum value on the symmetrical curve (see Fig. 4). The apparent region ratio of the serum-derived residues was defined as the ratio between the area of the apparent residue and the area of the entire acquired image; ImageJ (NIH, Bethesda, MD) was used for image processing.

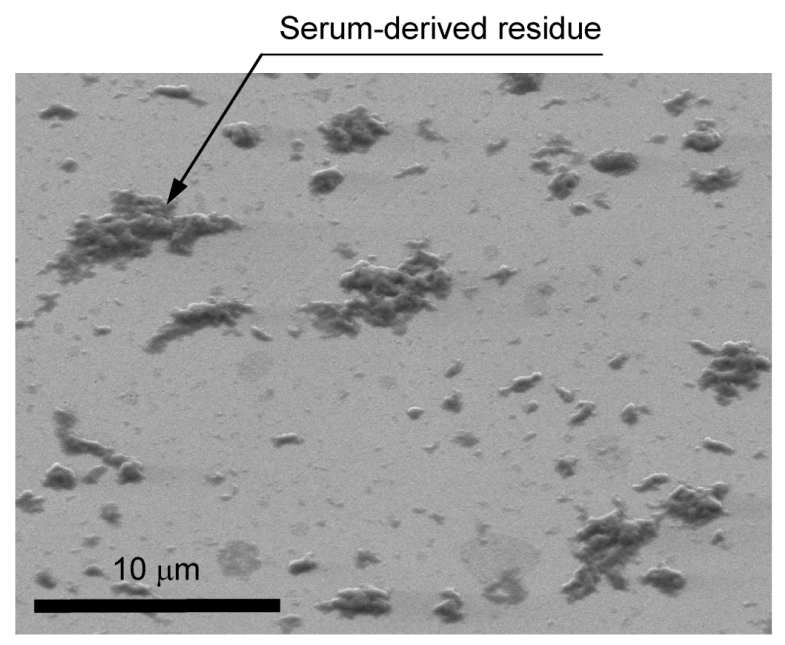

Fig. 3 Scanning electron micrograph of Co-28Cr6 Mo alloy surface after friction test

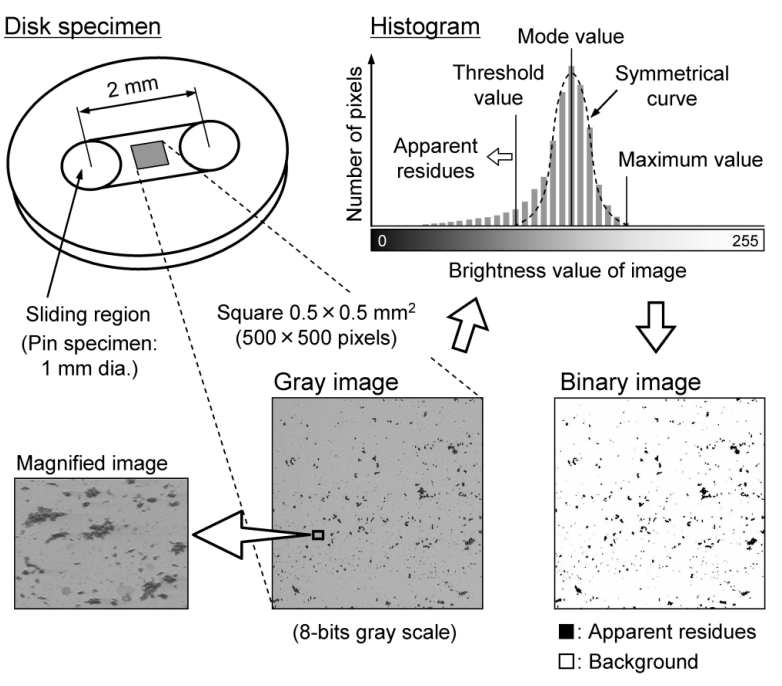

Fig. 4 Quantification procedure for serum-derived residues formed on $\mathrm{Co}-28 \mathrm{Cr}-6 \mathrm{Mo}$ alloy surface

\subsection{Statistical analysis}

The effects of vitamin $\mathrm{E}$ addition on the serum-derived residues on the Co-28Cr-6Mo alloy surface were analyzed using one-way ANOVA followed by the Tukey test for post hoc comparisons.

\section{Results}

The frictional performance of vitamin E-blended UHMWPE in serum lubricant tended to be similar to that of virgin UHMWPE under $3 \mathrm{MPa}$ loading, as shown in Fig. 5. Under $30 \mathrm{MPa}$ loading, the frictional performance of vitamin E-blended UHMWPE in serum lubricant exhibited three features that were not observed for virgin UHMWPE, as shown in Fig. 6. Firstly, the friction force was lower in the initial stage of friction tests (sliding distance: $0-2 \mathrm{~m}$ ); secondly, the variability of the friction force was less in the transition process $(2-5 \mathrm{~m})$; and thirdly, friction force was significantly higher in the steady state sliding $(5-8 \mathrm{~m})$. The frictional performance of virgin UHMWPE in serum lubricant was altered by the application of vitamin $\mathrm{E}$ to the sliding surface, as shown in Fig. 7. The frictional performance of vitamin E-applied UHMWPE was similar to that of vitamin E-blended UHMWPE.

The apparent region ratio of serum-derived residues on the Co-28Cr-6Mo alloy surface after friction tests for the vitamin E-blended, vitamin E-applied and virgin UHMWPE pin specimens are shown in Fig. 8. For vitamin E-blended UHMWPE, a significantly larger amount of serum-derived residues were observed in comparison with virgin UHMWPE. For virgin UHMWPE, a trend of increasing the serum-derived residues was observed with the application of vitamin $E$ to the sliding surface. 


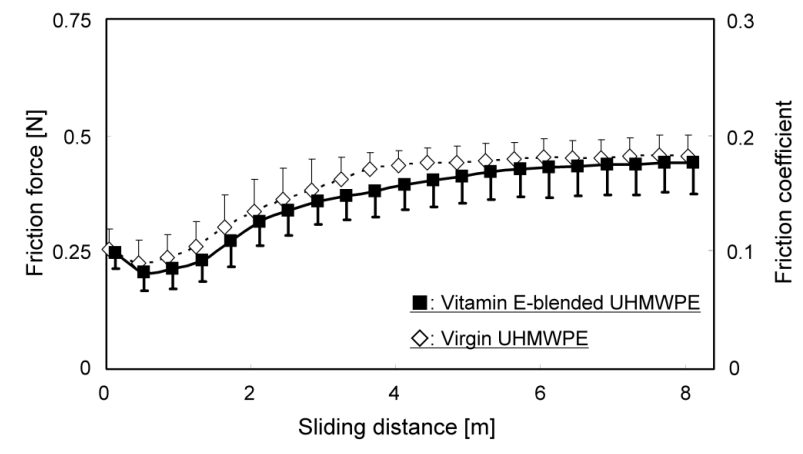

Fig. 5 Frictional performance of vitamin E-blended UHMWPE and virgin UHMWPE in serum lubricant under $3 \mathrm{MPa}$ loading. Data represent mean $\pm \operatorname{SD}(n=6)$

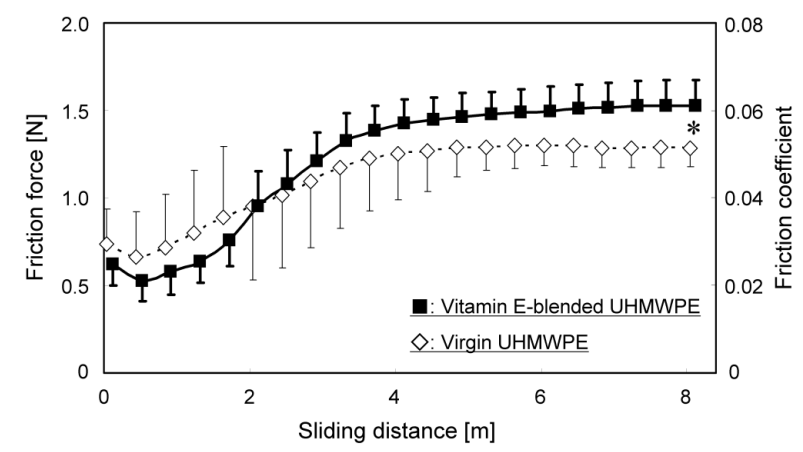

Fig. 6 Frictional performance of vitamin E-blended UHMWPE and virgin UHMWPE in serum lubricant under $30 \mathrm{MPa}$ loading. Data represent mean $\pm \mathrm{SD}$. Asterisk indicates a statistically significant difference $(* ; \mathrm{P}<0.01$, $\mathrm{n}=6$, Student's t-test)

\section{Discussion}

The frictional performance of vitamin E-blended UHMWPE in serum lubricant differed from that of virgin UHMWPE under $30 \mathrm{MPa}$ loading, while there was little difference under $3 \mathrm{MPa}$ loading. These results suggest that the effects of vitamin $\mathrm{E}$ on the frictional performance of UHMWPE in serum lubricant depend on the compressive load on the sliding surface. One explanation for these results is that the bulk properties of UHMWPE were altered by vitamin E blending. Two phenomena have been suggested to change the bulk properties of UHMWPE. Firstly, vitamin $\mathrm{E}$ in the amorphous phase of UHMWPE might decrease strain-induced crystallization and increase the strain-induced orientation of molecular chains in the amorphous phase ${ }^{9)}$. Secondly, the prevention of oxidation chain reactions (radical reactions) by vitamin E might affect the performances of $\gamma$-irradiated UHMWPE $^{1)}$. However, the materials used in this study were not subjected to $\gamma$-irradiation, and the mechanical properties of the non-oxidized UHMWPE with and without vitamin $\mathrm{E}$ are almost the same ${ }^{12)}$.

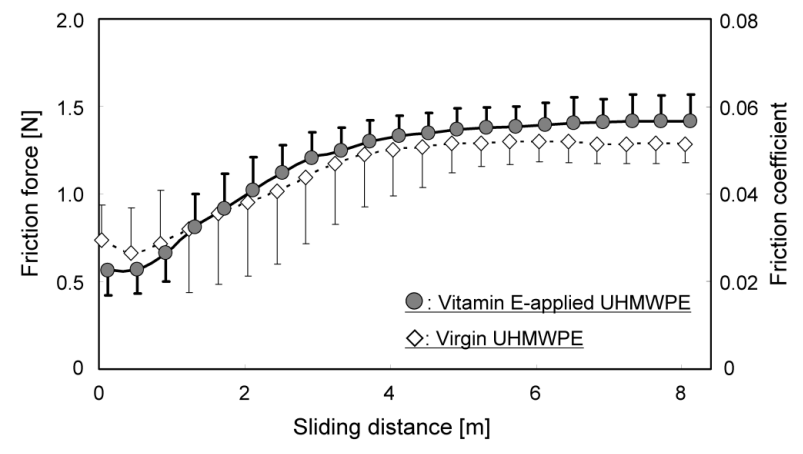

Fig. 7 Frictional performance of vitamin E-applied UHMWPE and virgin UHMWPE in serum lubricant under $30 \mathrm{MPa}$ loading. Data represent mean $\pm S D,(n=6)$

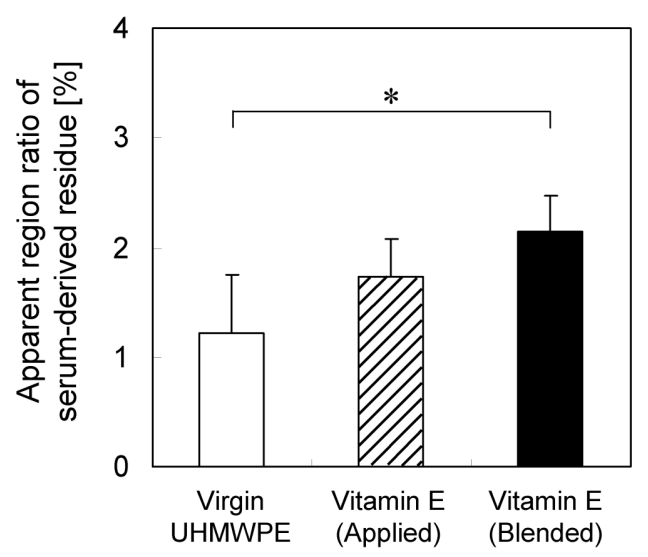

Fig. 8 Apparent region ratio of serum-derived residues on $\mathrm{Co}-28 \mathrm{Cr}-6 \mathrm{Mo}$ alloy surface after friction tests under $30 \mathrm{MPa}$ loading for each type of UHMWPE specimen. Data represent mean \pm SD. Asterisk indicates a statistically significant difference $(* ; \mathrm{P}<0.05, \mathrm{n}=5)$

Therefore, we have investigated an alternative mechanism, that is, vitamin E leaching from inside of vitamin E-blended UHMWPE under compressive load. We have found that blended vitamin $\mathrm{E}$ migrates to the surface of UHMWPE under compressive load, and the amount is positively correlated with the applied load ${ }^{10)}$. The frictional performance of the vitamin E-blended UHMWPE at the initial stage of the frictional test is likely affected by the presence of vitamin $\mathrm{E}$ on the sliding surface. The friction force of vitamin E-blended UHMWPE tended to be lower in the first $2 \mathrm{~m}$ and higher in the steady state $(5-8 \mathrm{~m})$, compared with that for virgin UHMWPE. Our results also show that the friction force of virgin UHMWPE in the first $1 \mathrm{~m}$ is decreased by the application of vitamin $\mathrm{E}$ to the sliding surface. Although the application of vitamin $\mathrm{E}$ to virgin UHMWPE at the onset of the friction test differs from the leaching of vitamin $\mathrm{E}$ from inside of vitamin E-blended UHMWPE during the friction test, this result 
suggests that the friction force of UHMWPE in the initial stage of friction tests is decreased by the presence of vitamin $\mathrm{E}$ on the sliding surface. However, it is unlikely that vitamin $\mathrm{E}$ leaches from inside of vitamin E-blended UHMWPE over the entire testing period, and vitamin $\mathrm{E}$ that leaches during the initial stage is considered to disperse gradually into the lubricant as the sliding distance increases. Another feature of the frictional test on the vitamin E-blended UHMWPE is that the frictional force exhibited less variability in the first $5 \mathrm{~m}$, and the amount of serum-derived residues on the Co-28Cr-6Mo alloy surface after friction tests was significantly larger than that on the virgin UHMWPE surface. Since the friction force and the serum-derived residues were increased by the application of vitamin $\mathrm{E}$ to the sliding surface of virgin UHMWPE, this finding suggests that the significantly higher friction force of vitamin E-blended UHMWPE in the steady state of the friction test can be attributed to the substances generated by the interaction between vitamin $\mathrm{E}$ and serum proteins on the sliding surface. However, it would be thought that the leached-vitamin E-mediated interaction with serum proteins less affected the steady state of friction under $3 \mathrm{MPa}$ loading, compared to that under $30 \mathrm{MPa}$ loading. Moreover, this interaction can be considered as one of the possible factors that lead to lower variability of the friction force of vitamin E-blended UHMWPE in the transition process.

Among the interactions between vitamin $\mathrm{E}$ and proteins, it is well known that the intricate secondary and tertiary structures of globular proteins become unfolded in aqueous solution via interaction with oil ${ }^{13)}$. In relation to friction, it has been suggested that unfolded proteins preferentially adsorb onto hydrophobic surfaces and/or other unfolded proteins due to strong hydrophobic interactions, and consequently efficient hydration of the frictional interface is decreased ${ }^{14)}$. However, the effects of serum proteins on the friction and wear performance of UHMWPE encompasses complex phenomena affected by many mechanisms and factors, for example, the extremely high pressure at the real contact area; thus, further experiments are necessary to elucidate these effects.

In the present study, the friction force of vitamin E-blended UHMWPE had a lower initial value and a higher value in the steady state, compared with that of virgin UHMWPE. In actual walking cycles, however, contact stress over $30 \mathrm{MPa}$ on the UHMWPE tibial component occurs only for short time intervals, and serum proteins are subsequently metabolized. Our results suggest that the friction force after the high compressive contact between UHMWPE and Co-28Cr-6Mo alloy is decreased temporarily by the presence of vitamin $\mathrm{E}$ on the sliding surface. This mechanism is a possibly contributes to the improved wear resistance of vitamin E-blended UHMWPE observed in knee simulator testing.

\section{Conclusion}

The load-dependent frictional performance of vitamin E-blended UHMWPE in serum lubricant was examined. The friction force of vitamin E-blended UHMWPE had a lower initial value and a higher value in the steady state, and the variability of friction force in the transition process was relatively small. Frictional performance similar to that of vitamin E-blended UHMWPE was observed as a result of applying vitamin $\mathrm{E}$ to the sliding surface of virgin UHMWPE. These results suggest that the frictional performance of vitamin E-blended UHMWPE in serum lubricant is affected by the presence of vitamin $\mathrm{E}$ on the sliding surface under high compressive load.

\section{Acknowledgments}

UHMWPE blocks (vitamin E-blended, virgin) and the Co-28Cr-6Mo alloy ingot were supplied by Nakashima Medical Corporation. UHMWPE pin specimens were machined by Yasojima Proceed Corporation. Co-28Cr-6Mo alloy disk specimens were machined and polished by Sanki Corporation. The authors are grateful to Mr. Sadamu Kinoshita, Graduate School of Engineering, Kyoto University, for SEM observation.

\section{References}

[1] Shibata, N., Kurtz, S. M. and Tomita, N., "Recent Advances of Mechanical Performance and Oxidation Stability in Ultrahigh Molecular Weight Polyethylene for Total Joint Replacement: Highly Crosslinked and $\alpha$-Tocopherol Doped," Journal of Biomechanical Science and Engineering, 1, 2006, 107-123.

[2] Kurtz, S. M., Muratoglu, O. K., Evans, M. and Edidin, A. A., "Advances in the Processing, Sterilization, and Crosslinking of Ultra-High Molecular Weight Polyethylene for Total Joint Arthroplasty," Biomaterials, 20, 1999, 1659-1688.

[3] Walker, P. S., Blunn, G. W. and Lilley, P. A., "Wear Testing of Materials and Surfaces for Total Knee Replacement," Journal of Biomedical Materials Research, 33, 1996, 159-175.

[4] Tomita, N., Kitakura, T., Onmori, N., Ikada, Y. and Aoyama, E., "Prevention of Fatigue Cracks in Ultrahigh Molecular Weight Polyethylene Joint Components by the Addition of Vitamin E," Journal of Biomedical Materials Research, 48, 1999, 474-478.

[5] Ingham, E. and Fisher, J., "The Role of Macrophages in Osteolysis of Total Joint Replacement," Biomaterials, 26, 2005, 1271-1286.

[6] Archibeck, M. J., Jacobs, J. J., Roebuck, K. A. and Glant, T. T., "The Basic Science of Periprosthetic Osteolysis," Journal of Bone and Joint Surgery (American), 82, 2000, 1478-1489. 
[7] Teramura, S., Sakoda, H., Terao, T., Endo, M. M., Fujiwara, K. and Tomita, N., "Reduction of Wear Volume from Ultrahigh Molecular Weight Polyethylene Knee Components by the Addition of Vitamin E," Journal of Orthopaedic Research, 26, 2008, 460-464.

[8] Teramura, S., Russell, S., Ingham, E., Fisher, J., Tomita, N., Fujiwara, K. and Tipper, J. L., "Reduced Biological Response to Wear Particles from UHMWPE Containing Vitamin E," Proc. 55th Annual Meeting of Orthopaedic Research Society, 2009, 2377.

[9] Okubo, Y., Teramura, S., Niwa, Y., Ibaraki, K., Murata, K., Hyon, S. H., Pezzotti, G. and Tomita, N., "Strain-Induced Crystallization and Orientation of Vitamin E-Blended Ultrahigh Molecular Weight Polyethylene," Bio-Medical Materials and Engineering, in press.

[10] Okubo, Y., Mori, S., Yamamoto, K. and Tomita, N., "Confirmation of Pressure-Induced Leaching of Vitamin E from inside of Ultrahigh Molecular Weight Polyethylene," Journal of Biomechanical Science and Engineering, in press.
[11] Okubo, Y., Mori, S., Yamamoto, K., Hamada, D., Kohno, H., Fujiwara, K., Hashimoto, M., Ikeuchi, K. and Tomita, N., "Mechanical Interaction between Vitamin E-Containing Ultrahigh Molecular Weight Polyethylene and Co-28Cr-6Mo Alloy in Water," Journal of Biomechanical Science and Engineering, 4, 2009, 166-173.

[12] Sakuramoto, I., Mori, A., Nagata, K., Tomita, N., Kuramoto, K. and Kawano, S., "The Effects of Oxidative Degradation on Mechanical Properties of UHMWPE for Artificial Knee Joint," Journal of the Japan Society of Mechanical Engineers A, 67, 2001, 1702-1709 (in Japanese).

[13] Meng, G., Chan, J. C. K., Rousseau, D. and Li-Chan, E. C. Y., "Study of Protein-Lipid Interactions at the Bovine Serum Albumin/Oil Interface by Raman Microspectroscopy," Journal of Agricultural and Food Chemistry, 53, 2005, 845-852.

[14] Heuberger, M. P., Widmer, M. R., Zobeley, E., Glockshuber, R. and Spencer, N. D., "Protein-Mediated Boundary Lubrication in Arthroplasty,” Biomaterials, 26, 2005, 1165-1173. 